\title{
IMPLICAÇÕES NEUROPSICOPEDAGÓGICAS NA COMPREENSÃO CLÍNICA DO TRANSTORNO DE DÉFICIT DE ATENÇÃO/HIPERATIVIDADE (TDAH) EM CRIANÇAS
}

\author{
NEUROPSYCHOPEDAGOGICAL IMPLICATIONS \\ FOR CLINICAL UNDERSTANDING ATTENTION DEFICIT / HYPERACTIVITY \\ DISORDER (ADHD) IN CHILDREN \\ IMPLICACIONES NEUROPSICOPEDAGÓGICAS PARA LA \\ COMPRENSIÓN CLÍNICA TRASTORNO POR DÉFICIT DE ATENCIÓN E \\ HIPERACTIVIDAD (TDAH) EN NINOS
}

Guilherme Faquim Simão

E-mail: guilhermefaquim@hotmail.com

Daniele Morgenstern Aimi E-mail: danielefm@gmail.com

Thiago Henrique Barnabé Correa E-mail: correa.uftm@,gmail.com

\begin{abstract}
RESUMO
A Neuropsicopedagogia surge do entrelaçamento entre as Neurociências, a Psicologia Cognitiva e a Educação. Tal ciência tem por objetivo principal o estudo entre a relação do sistema nervoso e a aprendizagem humana, prezando por uma integração biopsicossocial. O Transtorno de Déficit de Atenção/Hiperatividade (TDAH) é hoje um dos acometimentos que mais atinge crianças/adolescentes e adultos no mundo, sendo a principal causa de encaminhamento ao neuropediatra. É definido como padrão persistente de desatenção e/ou hiperatividade mais frequente, e em graus maiores ao observado em indivíduos com nível equivalente de desenvolvimento. Esta pesquisa tem por objetivo compreender de que maneira a Neuropsicopedagogia Clínica pode auxiliar na compreensão e possível intervenção no TDAH. Metodologicamente, adotou-se a modalidade de pesquisa exploratória através de levantamento bibliográfico e documental que abarque os eixos/descritores Neuropsicopedagogia e TDAH de maneira pormenorizada, intuindo correlacioná-los ao longo do artigo. Concluise, assim, que a Neuropsicopedagogia Clínica através da aplicação de instrumentos próprios, planejamento e intervenção neuropsicopedagógica poderia contribuir de forma significativa na melhoria dos sintomas de tal transtorno, ressoando na melhoria da qualidade de vida do indivíduo. Evidencia-se, ainda, que mais pesquisas na área Neuropsicopedagógica Clínica atreladas ao TDAH precisam ser realizadas, a fim de validar melhor sua efetividade, principalmente no que tange a prática, os procedimentos técnicos e as respostas às intervenções.
\end{abstract}

PALAVRAS-CHAVE: Neuropsicopedagogia. TDAH. Estratégias de intervenção.

\section{ABSTRACT}

Neuropsychopedagogy arises from the intertwining of Neurosciences, Cognitive Psychology and Education. This science has as its main objective the study between the relationship of the nervous system and human learning, valuing a biopsychosocial integration. Attention Deficit/Hyperactivity Disorder (ADHD) is currently one of the affections that most affects children/adolescents and adults in the world, being the main cause of referral to the neuropediatrician. It is defined as a more frequent persistent pattern of inattention and/or hyperactivity, and in greater degrees than observed in individuals with an equivalent level of development. This research aims to 


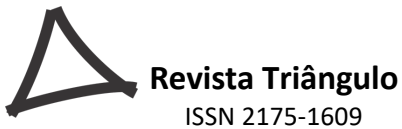

ISSN 2175-1609

understand how Clinical Neuropsychopedagogy can help in understanding and possible intervention in ADHD. Methodologically, the exploratory research modality was adopted through a bibliographic and documental survey that encompasses the axes/descriptors Neuropsychopedagogy and ADHD in a detailed manner, aiming to correlate them throughout the article. It is concluded, therefore, that Clinical Neuropsychopedagogy, through the application of its own instruments, planning and neuropsychopedagogical intervention could significantly contribute to the improvement of the symptoms of such disorder, resonating with the improvement of the individual's quality of life. It is also evident that more research in the Clinical Neuropsychopedagogical area linked to ADHD needs to be carried out in order to better validate its effectiveness, especially with regard to practice, technical procedures and responses to interventions.

KEYWORDS: Neuropsichopedagogy. ADHD. Intervention Strategies.

\section{RESUMEN}

La neuropsicopedagogía surge del entrelazamiento de las neurociencias, la psicología cognitiva y la educación. Esta ciencia tiene como principal objetivo el estudio entre la relación del sistema nervioso y el aprendizaje humano, valorando una integración biopsicosocial. El Trastorno por Déficit de Atención / Hiperactividad (TDAH) es actualmente una de las afecciones que más afecta a niños / adolescentes y adultos en el mundo, siendo la principal causa de derivación al neuropediatra. Se define como un patrón persistente más frecuente de inatención y / o hiperactividad, y en mayor grado que el observado en individuos con un nivel de desarrollo equivalente. Esta investigación tiene como objetivo comprender cómo la neuropsicopedagogía clínica puede ayudar en la comprensión y la posible intervención en el TDAH. Metodológicamente, se adoptó la modalidad de investigación exploratoria a través de una encuesta bibliográfica y documental que cubre los ejes / descriptores Neuropsicopedagogía y TDAH de manera detallada, con el objetivo de correlacionarlos a lo largo del artículo. Se concluye, por tanto, que la Neuropsicopedagogía Clínica, mediante la aplicación de sus propios instrumentos, la planificación y la intervención neuropsicopedagógica podrían contribuir significativamente a la mejora de los síntomas de dicho trastorno, resonando con la mejora de la calidad de vida del individuo. También es evidente que es necesario realizar más investigaciones en el área Clínica Neuropsicopedagógica vinculada al TDAH para validar mejor su efectividad, especialmente en lo que respecta a la práctica, los procedimientos técnicos y las respuestas a las intervenciones.

PALABRAS-CLAVE: Neuropsicopedagogía. TDAH. Estrategias de intervención.

\section{INTRODUÇÃO}

As bases teóricas que irão balizar a formação e atuação dos profissionais da Neuropsicopedagogia advêm das Neurociências e da própria Educação, como sinaliza Russo (2015). Entretanto, outras fontes, além das supracitadas, serão também acessadas, já que a Neuropsicopedagogia bebe também em matrizes da Psicologia Cognitiva e da Pedagogia ao fazer uma interlocução entre esses distintos saberes para abarcar melhor as dissemelhantes constituições humanas. Ao pensar no indivíduo do século XXI é impossível não o visualizar como um ser biopsicossocial, cujas dimensões estão inteiramente imbricadas. Dessa forma, a Neuropsicopedagogia tem como objeto principal o estudo entre a relação do sistema nervoso e a aprendizagem humana, primando por uma reintegração social, pessoal e escolar, não se 
esquecendo também dos fatores externos e ambientais que muito contribuem para que o processo de ensino e aprendizagem ocorra ou não.

Como apontado por Fonseca (2014), a Neuropsicopedagogia reúne e integra “os estudos do desenvolvimento, das estruturas, das funções e das disfunções do cérebro, ao mesmo tempo que estuda os processos psicocognitivos responsáveis pela aprendizagem e os processos psicopedagógicos responsáveis pelo ensino" (FONSECA, 2014, p.236).

Dessa maneira, as Neurociências, a Psicologia Cognitiva e a Pedagogia se interrelacionam e se constituem em ancoradouros da ciência Neuropsicopedagógica para auxiliar na compreensão e possível intervenção de dificuldades e transtornos da aprendizagem, sendo um deles o Transtorno de Déficit de Atenção/Hiperatividade (TDAH).

A aprendizagem tem lugar privilegiado e cativo em nossa sociedade, uma vez que todos precisam cumprir (em diferentes graus) essa tarefa: aprender, ou pelo menos é assim que esse imperativo da aprendizagem é posto, como aponta Filho e Bridi (2016). Ao atender tal imperativo, depreende-se que uma maioria tenha um nível de aprendizagem análogo e sistematizado, inclusive concernente ao comportamento. Dessa maneira, aqueles que acabam por não corresponder ao que lhes é imputado são vistos como atípicos, dando margem para a constituição do campo das chamadas "dificuldades de aprendizagem" e transtornos da aprendizagem.

O TDAH é um distúrbio neurobiológico crônico que se caracteriza por desatenção, desassossego e impulsividade, apresentando-se com grande frequência na atualidade. Acomete, principalmente, crianças e adolescentes, mas também adultos e pode causar prejuízos ao longo de toda vida do indivíduo, agravando as questões sociais e de aprendizagem. É importante ressaltar que transtornos relacionados à atenção nem sempre estão associados à hiperatividade. Sua relação com as dificuldades de aprendizagem é a principal causa de consultas neuropediátricas, sejam encaminhadas pela escola ou por demais profissionais que corroboram no rastreio do transtorno.

Pessoas acometidas por esse transtorno têm alto risco para mau rendimento escolar, dificuldades de relacionamento, atos delinquentes, acidentes de automóvel, uso de álcool e outras drogas, além de dificuldades no âmbito profissional (ROTTA, 2016).

Como aporte metodológico para estruturação desse artigo, adotou-se a modalidade de pesquisa exploratória que não requer a formulação de hipóteses a serem testadas, restringindose a definir objetivos e buscar mais informações sobre determinado assunto específico, ou que 
ainda tal temática tenha sido pouco explorada, como é o caso do presente artigo. Normalmente esse método exploratório é o passo inicial para um projeto de pesquisa, e posterior desdobramento em hipóteses a serem mais bem esclarecidas ao longo da realização do mesmo (CERVO; BERVIAN; DA SILVA, 2007).

Tem-se ainda, por objetivo dentro da pesquisa exploratória, desenvolver uma visão mais generalista sobre determinado objeto que tenha por desfecho uma problemática mais esclarecida, e que resulta em hipóteses pesquisáveis em estudos posteriores, como reitera Gil (1999).

Primeiramente foram buscados artigos na biblioteca virtual e indexadora Scientific Eletronic Library Online (SciELO), por se tratar de uma plataforma cujos artigos passam por uma criteriosa avaliação teórico-metodológica. Ao buscar pelo descritor "neuropsicopedagogia", nenhuma referência foi encontrada. Já na plataforma Google Acadêmico, que também segue crivos de seleção parecidos aos da SciELO, foram achados 25 resultados com o mesmo descritor, sendo 22 artigos e 3 livros a serem adquiridos, e por isso foram excluídos. Desses 22 artigos encontrados, ao afunilar uma nova busca juntando as palavras-chave "neuropsicopedagogia" e "TDAH", nenhum artigo foi encontrado.

Dessa maneira, pela dificuldade em buscar produções/referências que abarcassem os dois eixos principais da pesquisa (Neuropsicopedagogia e TDAH) de forma correlacionada, optou-se por uma pesquisa que buscasse explorar de maneira mais pormenorizada cada um desses eixos-chave na tentativa de interseccioná-los ao longo da construção do artigo. Literaturas que contemplem áreas correlatas à Neuropsicopedagogia como as Neurociências, a Neuropsicologia, a Psicopedagogia, entre outras, também serão utilizadas ao longo do artigo para auxiliar a construção de um arcabouço teórico mais rico.

Quanto ao grau de relevância da pesquisa, acredita-se que este seja um tema altamente pertinente para profissionais de diversas áreas (educação, saúde, entre outras) por se tratar de uma temática transdisciplinar, assim como para pessoas que se interessam pela temática. Outro fator valoroso na exploração do assunto em questão se dá pela própria ciência Neuropsicopedagógica que é relativamente nova e ainda carece de maiores explanações. Por fim, discorre-se também que por estar finalizando uma pós-graduação na referida área de pesquisa desse artigo, o autor principal tem por intento pessoal compreender de que forma tal ciência poderá balizar sua prática, a compreensão do transtorno e o planejamento das possíveis intervenções. 


\section{CONSTITUIÇÃO DA NEUROPSICOPEDAGOGIA NO BRASIL E A REGULAÇÃO DA ATUAÇÃO CLÍNICA}

São bem recentes os registros históricos sobre a constituição e disseminação da Neuropsicopedagogia no Brasil, surgindo na primeira década do século XXI em cursos de Pedagogia no sul do país. Como assuntos-chave na disciplina, eram passados conteúdos que abarcassem conceitos sobre a estruturação cerebral, o desenvolvimento neuropsicológico e a plasticidade do cérebro, por exemplo.

No decorrer do curso, ao se constatar que o processo de aprender não seria uma construção estanque, e que vários fatores estão associados ao aprendizado, foi pensado um curso de pós-graduação que contemplasse não somente a teoria, mas também a prática interventiva em questões neuropsicopedagógicas. Assim, em 2009, o curso de Pós-graduação em Neuropsicopedagogia foi instituído pela Faculdade Ciências, Educação, Saúde, Pesquisa e Extensão (Censupeg).

Segundo a Sociedade Brasileira de Neuropsicopedagogia (SBNPp), a formação do Neuropsicopedagogo se dá através do curso de pós-graduação (lato sensu) com a titulação mínima certificada de Neuropsicopedagogia. Seu principal intuito é construir uma representatividade social que possa promover a ascensão da nova profissão no cenário nacional, além de normatizar e codificar as diretrizes técnico-científicas na prática profissional. De acordo com a SBNPp, o profissional Neuropsicopedagogo pode atuar em duas áreas distintas: a Institucional e a Clínica, sendo essa última objeto de estudo desse presente artigo. Para tanto, deverá seguir as normativas estabelecidas pela SBNPp, que sinalizam:

Art. 30 - Ao Neuropsicopedagogo com formação clínica, conforme descrito no Capítulo $\mathrm{V}$, fica delimitada sua atuação com atendimentos neuropsicopedagógicos individualizados em "setting" adequado, como consultório particular, espaço de atendimento, posto de saúde, terceiro setor. Os atendimentos em local escolar e hospitalar devem acontecer de forma individual e em local adequado (RUSSO, 2015, p.16).

Está dentro do escopo de atuação do Neuropsicopedagogo Clínico a observação, identificação e análise do ambiente escolar no que tange o desenvolvimento humano em áreas motoras, cognitivas e comportamentais (RUSSO, 2015). Aponta, ainda, a avaliação, intervenção e acompanhamento do indivíduo com dificuldades de aprendizagem, transtornos, 
altas habilidades e síndromes que causem prejuízo em alguma esfera na parte escolar e/ou social através da utilização de protocolos e instrumentos de avaliação/reabilitação devidamente validados. Somado ao exposto é importante enfatizar que não cabe a este profissional instituir prescrições farmacológicas. Sua atuação é importante no trabalho multiprofissional de modo a ajudar o indivíduo a desempenhar suas atividades cotidianas da melhor forma possível.

Para Russo (2015) as semelhanças entre a Neuropsicopedagogia Clínica, a Psicopedagogia e a Neuropsicologia residem na origem multiprofissional, inter e transdisciplinar, além do estudo do desenvolvimento humano e dos processos de ensino e aprendizagem, sejam eles em condições normais ou não. Sendo assim, importante salientar que cada ciência possui finalidades de atuação próprias, inclusive no que tange os testes e protocolos aplicados.

Como ciências basilares para a constituição da Neuropsicopedagogia, as Neurociências, a Psicologia Cognitiva e a Pedagogia se entrelaçam para auxiliar o profissional Neuropsicopedagogo na construção teórica e prática de questões relacionadas à aprendizagem. Vários estudos sinalizam que não basta o professor conhecer apenas os conceitos acerca do sistema nervoso e o processo de aprendizagem, precisa também compreender como intervir nesse processo. A ele impende, de forma conjunta, conhecer o funcionamento cognitivo e comportamental do sujeito que aprende (FÜLLE et al., 2018). Dessa forma:

\footnotetext{
Saber como o cérebro evoluiu, evolui e funciona é determinante para o sucesso não só na aprendizagem como no ensino, o chamado processo ensino-aprendizagem, que consubstancia a característica única da espécie humana de transmitir a cultura intergeracionalmente, ou seja, entre seres maturos e experientes e seres imaturos e inexperientes (FONSECA, 2014, p.2).
}

A Neurociência traz para o contexto educacional um caráter mais científico e aprofundado sobre a aprendizagem, explicando a estrutura e o funcionamento neural. Tais conhecimentos gerados pelas neurociências têm contribuído para um progresso na educação, melhoria na qualidade de vida e possibilitado uma maior eficiência na aprendizagem (COSENZA; GUERRA, 2011). Para os autores, saber simplesmente como o cérebro aprende não é suficiente para que a "mágica" do ensinar e aprender ocorra, mas que seria possível correlacionar os fatores neurobiológicos com assuntos pedagógicos. Assim, para eles, a maioria dos profissionais ligados à educação não possuem uma formação para o atendimento das demandas da aprendizagem, e acreditam que tais profissionais poderiam se beneficiar de 
conhecimentos neurocientíficos para uma melhor abordagem das questões escolares e possíveis intervenções.

\section{TDAH: ASPECTOS HISTÓRICOS, NEUROPSICOLÓGICOS E CLÍNICOS}

Desde o final do século XIX, pesquisadores e teóricos se debruçam em compreender o porquê algumas crianças apresentavam baixo rendimento escolar e comportamentos "atípicos", demostrando que a situação encontrada na atualidade não é tão recente assim. Dessa maneira, enseja-se percorrer a construção histórica e a constituição clínica do TDAH.

\section{Construção histórica}

Rotta (2016) preconiza que, em 1896, Bourneville descreveu comportamentos que se caracterizavam como "uma instabilidade que seria uma ruptura do equilíbrio entre os impulsos e as inibições (2016, p. 274)", mas que, somente em 1900, um teórico chamado Still descreveu um quadro clínico com características de déficit de atenção e hiperatividade. Binet e seu colaborador, Simon, estiveram envolvidos em estudos sobre o diagnóstico do chamado retardo mental, atualmente chamado de deficiência intelectual, e a forma de educação de crianças “atípicas" para a época, tecendo construções que seriam amplamente desenvolvidas posteriormente por outros teóricos.

Em 1905, Binet e Simon apresentam no Congresso Internacional de Psicologia, em Roma, os resultados de um trabalho de extrema relevância e que, em 1908, torna-se uma escala de análise da inteligência através de um método denominado de Binet-Simon (1908 apud TEIXEIRA, 2019). Tais testes permitiram estabelecer curvas de desenvolvimento psicológico da criança, diagnosticar as variações da inteligência, além de aspectos perceptivos, motores e afetivos. Tais autores acabam identificando três tipos diferentes de crianças que frequentavam as escolas especiais: os deficientes mentais, os instáveis e um grupo misto, formado por deficientes com problemas de comportamento. O grupo denominado instável, ainda que parecessem possuir deficiência, apresentavam bom rendimento intelectual na avaliação psicológica (ROTTA, 2016).

Wallon publica, em 1925, seu livro intitulado L'Enfant turbulent, onde irá caracterizar uma constituição específica de instabilidade ao definir que alterações sensoriais também se 
manifestavam na área motora. Em 1947, os autores Strauss e Lehtine descrevem uma síndrome que se caracterizava pelas irregularidades do aprendizado, função psíquica, comportamento e da motricidade. Tal caracterização levava em conta que por se ter um cérebro com dificuldades em controlar tamanha reatividade (hipervigilante), quando exposto aos estímulos externos, não seria possível controlar seus impulsos, tornando-se instável, desatento e com a atenção desviada em situações que, para as demais crianças, não seria uma problemática (ROTTA, 2016).

O termo utilizado na época para situações similares à descrita acima seria: lesão cerebral mínima (LCM), sendo substituído, posteriormente, por disfunção cerebral mínima (DCM), como sinaliza Clements (1966) em seu artigo Minimal Brain Dysfunction in Children. Adiante, o autor classificará tais disfunções como "déficit de atenção", e em 1975 passará a figurar realmente como um transtorno na Classificação Internacional de Doenças (CID-9), demarcado, essencialmente, por um grave déficit na atenção.

Em 1980, o Manual Diagnóstico e Estatístico de Transtornos Mentais (DSM-III) passou a conceituar esse acometimento psíquico de "transtorno de déficit de atenção com ou sem hiperatividade", sendo, a posteriori, substituído pelo termo "distúrbio de déficit de atenção e hiperatividade, no DSM-III-R (revisão do DSM-III). Já em 1994, no DSM-IV, o transtorno foi melhor delineado, e incluíram além da desatenção e hiperatividade, a impulsividade (ROTTA, 2016). Atualmente o TDAH (DSM-V, 2015; ROTTA, 2016) caracteriza-se por um nível inadequado de atenção quando comparada a idade de outros indivíduos, levando a distúrbios motores, perceptivos, cognitivos e comportamentais. Não necessariamente a criança precisa ter prejuízos nas três áreas, podendo ter sintomas combinados entre ser desatenta, hiperativa e/ou impulsiva.

\section{Aspectos neuropsicológicos e clínicos do TDAH}

A prevalência do referido transtorno, hodiernamente, seria de 3 a $30 \%$ em crianças no período escolar, e diminui à medida que a idade avança. Normalmente acomete mais meninos, na proporção de 2:1. Há ainda risco de que uma criança acometida por TDAH e que tenha mau rendimento escolar e dificuldades de relacionamento venha a desenvolver comportamentos delinquentes (PEREIRA et al., 2005). É sabido que entre 10 e 60\% dos casos de TDAH persistem na vida adulta, estimando-se uma prevalência de 0,3 a 2,5\% em adultos. Em relação ao gênero, no sexo masculino há um predomínio de características mais hiperativas e 
impulsivas; já no gênero feminino há um predomínio de desatenção como a expressão clínica mais relevante. Crianças com o transtorno possuem um maior risco de desenvolverem outras comorbidades relacionadas ao comportamento, como o Transtorno Opositivo Desafiador (TOD) e o Transtorno de Conduta.

Sua etiologia é multifatorial, com fatores genéticos e ambientais combinados, que podem ser divididos em pré (infecções maternas, intoxicações, irradiações etc.), peri (causas maternas, fetais e do parto) e pós-natais (nível socioeconômico, condições psicoafetivas, entre outros). O diagnóstico é definido por dados encontrados em diferentes fontes e situações, já que não existe um marcador biológico definido para todos os casos de TDAH, e que é classificado em leve, moderado e grave. Espera-se que a sintomatologia aconteça em diversos locais (não só a escola ou em casa) para que se chegue a um diagnóstico mais assertivo. A história clínica também é um fator de suma importância, a fim de se excluir fatores biológicos e síndromes que poderiam desenvolver comportamentos semelhantes.

De um viés neurológico, frequentemente, o TDAH está associado a distúrbios nas funções executivas e no córtex pré-frontal. Essas funções estão situadas entre os aspectos mais complexos da cognição, abarcando seleção e integração de informações atuais e previamente memorizadas, o planejamento, monitoramento e flexibilidade cognitiva (CANTIERE, 2014). Segundo a autora, a flexibilidade cognitiva aparece bem deficitária em crianças com o transtorno, podendo ser definida como a capacidade de raciocinar de diferentes maneiras na resolução de um problema e a habilidade de traçar novas rotas de raciocínio caso alguma intercorrência aconteça.

Sendo assim, sob o aspecto neuropsicológico, o déficit fundamental no referido transtorno é a incapacidade de modulação de respostas ao estímulo com impulsividade e desatenção. Por atenção, compreende-se como:

...tomada de posse pela mente de forma clara e vívida, de um entre os muitos objetos
ou cadeias de pensamento simultaneamente possíveis. A focalização, a concentração da
consciência, fazem parte da sua essência. Ela implica o afastamento de algumas coisas
de modo a que se possa lidar efetivamente com outras (JAMES, 1890 apud KANDEL,
2009, p.340).

A atenção é "uma parte da estrutura psíquica que permite selecionar e utilizar informações dentro de recursos mentais disponíveis" (FILHO et al., 2016, p.98). Os autores reiteram que os circuitos neuronais associados com o transtorno incluem o córtex pré-frontal, mas não somente, incluindo também os gânglios de base e o cerebelo. Segundo os autores, há 
relatos de alterações detectadas por meio de ressonância magnética que condizem com a disfunção pré-frontal com perda de assimetria normal do caudato, menor região frontal anterior direita, menor cerebelo e reversão da assimetria normal dos ventrículos laterais. Entretanto, será o critério clínico apontado pelos manuais psiquiátricos vigentes (CID-10/11 e DSM-V) que fará com que a base diagnóstica seja apontada.

Os autores postulam, ainda, que normalmente é na primeira infância que os pais identificam uma atividade motora excessiva. Entretanto, é no ensino fundamental que situações de desatenção vão ficando mais relevantes e prejudiciais para o desempenho da criança. Tais sintomas podem estabilizar ou apresentar agravamento na adolescência e chegar à vida adulta com sintomas graves de desatenção, mesmo se houver redução na hiperatividade.

Dentre os fatores propostos pelo DSM-V (2014) para diagnóstico do TDAH, em relação à desatenção, seis ou mais dos critérios sinalizados precisam ocorrer por pelo menos seis meses, gerando um impacto negativo nas esferas sociais, acadêmicas e/ou profissionais dos indivíduos (ROTTA, 2016); são eles:

a) Frequentemente não presta atenção ou cometo erros por descuido em locais distintos;

b) Constantemente tem dificuldades em manter a atenção em tarefas e atividades lúdicas;

c) Rotineiramente parece não ouvir quando alguém lhe dirige a palavra diretamente;

d) Frequentemente não segue instruções até o fim, culminando em dificuldades para terminar trabalhos escolares;

e) Constantemente tem dificuldade para organizar tarefas e atividades;

f) Rotineiramente reluta em se envolver em atividades que exijam maior esforço mental prolongado, não gosta delas ou as evita (em adolescentes e adultos tais questões podem estar relacionados com a dificuldade em preencher relatórios, formulários ou revisão de trabalhos longos);

g) Constantemente perde objetos ou coisas úteis para tarefas e atividades;

h) Frequentemente é distraído por estímulos externos (em adolescentes e adultos, podem incluir pensamentos não-relacionados);

i) Rotineiramente é esquecido em relação às atividades do dia a dia (realizar tarefas/obrigações, e com adolescentes e adultos pode estar relacionado a retornar ligações, pagar contas e manter uma agenda organizada). 
Já em relação à impulsividade/hiperatividade, a mesma prerrogativa de seis ou mais critérios durante pelos menos seis meses precisam ser atendidas, e que também apresentem prejuízos nas atividades sociais, acadêmicas e profissionais. Em adolescentes com mais 17 anos, pelo menos cinco sintomas serão necessários (ROTTA. 2016); são eles:

a) Frequentemente mexe mãos e/ou pés na cadeira;

b) Constantemente levanta-se da cadeira em situações em que se espera que permaneça sentado (sai do lugar na sala de aula, escritório e outros locais);

c) Rotineiramente corre ou sobe em objetos em situações em que tal comportamento é inadequado;

d) Constantemente está "agitado" em demasia;

e) Frequentemente fala demais e não consegue esperar a pergunta ser concluída, deixando escapar a resposta;

f) Rotineiramente interrompe ou se intromete em situações em que não há necessidade;

g) Vários sintomas de desatenção ou de hiperatividade/impulsividade estão presentes antes dos 12 anos;

h) Vários sintomas de hiperatividade/impulsividade estão presentes em mais de um ambiente;

i) Apresentam-se evidencias claras que os sintomas de desatenção, hiperatividade/impulsividade interferem no funcionamento social, acadêmico e social do indivíduo.

É de vital importância compreender que as crianças que aparecem com queixas de desatenção, comportamento hiperativo e impulsivo não necessariamente possuem TDAH, já que tais sintomas também aparecem em outros quadros. Dessa maneira, ressalta-se a importância de um diagnóstico diferencial cujos sintomas podem ser secundários a outros diagnósticos, como: epilepsia, déficit auditivo, déficit visual, lesões cerebrais, síndromes genéticas, doenças neurodegenerativas, doenças crônicas, doenças relacionadas à tireoide, distúrbios do sono, transtornos neuropsiquiátricos, transtornos do aprendizado, deficiência intelectual, além de fatores psicossociais (ROTTA, 2016).

\section{Anamnese, planejamento de avaliação e intervenção neuropsicopedagógica}


Para se planejar as possíveis intervenções em portadores de TDAH é necessário, de antemão, realizar uma anamnese criteriosa. Os dados do desenvolvimento desde a gestação até o parto, o desenvolvimento psicomotor, de linguagem, como vivencia a aprendizagem, como é seu comportamento na escola ou trabalho, sua relação com a família e seus iguais, também são critérios preponderantes na construção da anamnese. O primeiro ano de vida do indivíduo é de suma relevância (sono, choro, intercorrências no desenvolvimento da gestação, parto, entre outros). A história escolar do indivíduo é também indispensável para uma boa anamnese, desde o desempenho acadêmico, problemas de aprendizagem, comportamentais, até dificuldades na alfabetização, leitura, escrita e matemática. Manter contato com outros profissionais de saúde ou que estejam atuando no caso, caso o indivíduo esteja em outros acompanhamentos, faz-se necessário, já que ampliará a percepção sobre o funcionamento em outros locais/situações.

Por fim, importante evidenciar que o processo de identificação do TDAH deverá se utilizar da queixa principal que leva o paciente/responsáveis a buscar a avaliação, além da observação clínica, análise do material escolar (quando criança/adolescente), resultados de avaliações de habilidades sociais, e do contato com a equipe técnica-pedagógica da escola frequentada (em caso de escolares) (RUSSO, 2015; CARVALHO, 2018).

Os aspectos comportamentais também são substanciais para uma melhor compreensão do histórico do indivíduo, tais como: maneira como lida com a frustração, presença de trações de impulsividade, e possíveis problemas psicológicos. Em adolescentes/adultos, investigar, ainda, se há uso de substâncias como a maconha, já que a substância possui efeitos que causam lentidão visuomotora e sintomas de falta de atenção (ROTTA, 2016). O histórico dos pais também é de extrema relevância, já que, por se tratar de um transtorno com fatores genéticos, tais antecedentes podem ajudar no possível diagnóstico.

Finalizada a entrevista de anamnese, deve-se informar o objetivo da avaliação neuropsicopedagógica, a função de tal profissional nas intervenções escolares, previsão de número de sessões e a forma do encerramento. Importante salientar que o Neuropsicopedagogo Clínico, para proceder ao possível diagnóstico que será somado à avaliação médica, utiliza-se de dados oriundos das aplicações de testes e escalas normatizadas para a população brasileira. Tal formação não habilita o referido profissional a avaliar inteligência, transtornos de humor, transtornos psicóticos, transtornos alimentares, entro outros, como aponta Russo (2015). A seleção de instrumentos a serem utilizados na investigação é realizada de acordo com o objetivo 
da avaliação, prezando por materiais que não sejam vetados para seu uso e que forneçam parâmetros para analisar e avaliar dificuldades e facilidades de aprendizagem do indivíduo.

\section{Planejamento de avaliação neuropsicopedagógica}

A avaliação neuropsicopedagógica ocorre entre 3 a 6 sessões de 1 hora a 1 hora e 30 minutos, dependendo de cada caso. Após avaliação será acordado o trabalho de intervenção neuropsicopedagógica, que também irá variar em relação ao número de sessões. Tal plano de avaliação é construído a partir de perguntas ou hipóteses iniciais, definindo-se instrumentos necessários e de que maneira os utilizar. O paciente irá saber de todas as etapas do processo e objetivos elencados, sendo necessário, para um maior êxito da avaliação e posterior intervenção, um ambiente tranquilo e que motive o indivíduo a realizar tais atividades.

O primeiro momento de avaliação ocorre de maneira informal por meio da observação do comportamento do indivíduo/paciente durante toda a avaliação, além da maneira como interage e responde às atividades lúdicas e espontâneas. Tal análise é fundamental para um diagnóstico neuropsicopedagógico diferencial. Russo (2015) aponta que os resultados não se darão apenas analisando o resultado de testes, mas na integração desses resultados com os fatores de saúde, ambientais, emocionais, sociais e demais componentes que interferem na aprendizagem, como a atenção e as funções executivas no TDAH.

Depois de uma judiciosa anamnese e da construção do plano de avaliação, constrói-se o protocolo de avaliação neuropsicopedagógica, que é composto de instrumentos de leitura, escrita, aritmética, atenção, funções executivas, memória de aprendizagem e destreza motora. Em relação à atenção, a avaliação se dá por meio de instrumento validado e através da observação de atividades dirigidas e lúdicas, no que tange o foco atencional, tempo de concentração e se o indivíduo perdura atento mesmo quando acometido de estímulos distrativos (RUSSO, 2015).

Em relação à memória de aprendizagem, utilizam-se tarefas para avaliar o quanto o indivíduo é capaz de aprender uma informação nova (imediata) e o quanto consegue guardar a informação que foi aprendida (evocação tardia), além da memória de reconhecimento.

Ulteriormente à avaliação e à análise integrada de todos os dados, o Neuropsicopedagogo Clínico irá elaborar o relatório de avaliação neuropsicopedagógica, que contempla o final do processo, a síntese de conclusões e o prognóstico, a devolução ao paciente 
e/ou responsáveis, e os devidos encaminhamentos. Faz-se necessário, ademais, apresentar os resultados à escola, discutindo os possíveis ajustes metodológicos e interferências que podem estar dificultando a aprendizagem do indivíduo e potencializando os comportamentos hiperativos/disruptivos (RUSSO, 2015; CARVALHO, 2018).

\section{Intervenção neuropsicopedagógica}

Apesar de ser considerado o transtorno que mais afeta crianças/adolescentes na atualidade, grande parte desses indivíduos não são devidamente tratados em decorrência da resistência apresentada pelos pais em relação ao uso de medicamentos. Embora os medicamentos sejam indicados em alguns casos e apresentem efetividade a curto prazo, podem apresentar efeitos colaterais diversos, como dor de cabeça, palpitação, insônia, e inclusive atraso no crescimento (CANTIERE, 2014). Ainda segundo a autora, após suspensão dos medicamentos, os déficits cognitivos e comportamentais tendem a retornar.

Além de traçar um plano de avaliação e contribuir na elaboração de um diagnóstico e prognóstico do indivíduo/paciente, o Neuropsicopedagogo poderá também atuar na intervenção neuropsicopedagógica do sujeito, trabalhando com ele estratégias de aprendizagem variadas e definindo atividades que poderão facilitar e aprimorar seu processo ensino-aprendizagem. Como sinaliza Russo (2015), por serem conscientes e intencionadas, as estratégias de aprendizagem implicam em elaborar um Plano de Ação (intervenção) que se pautará nos objetivos a serem alcançados na fase inicial, intermediária e final do acompanhamento com o indivíduo. Segundo Portilho e Küster (2006) as estratégias de aprendizagem são operações mentais suscetíveis, ou seja, passíveis de serem modificadas, e que se classificam em cognitivas e metacognitivas.

As estratégias de aprendizagem cognitivas irão executar uma ação por meio de um conjunto de atividades ou técnicas que estarão a seu serviço; já as metacognitivas irão regular tudo o que está relacionado com o conhecimento, a fim de decidir quais estratégias serão utilizadas (quando e como), ademais de controlar, inclusive, a ação delas mesmas. São classificadas como: de conhecimento (pessoa, tarefa e estratégia) e de controle (planejamento, regulação e avaliação) (PORTILHO; KÜSTER, 2006; RUSSO, 2015).

Conhecida a classificação das formas de estratégias de aprendizagem que podem ser elencadas, faz-se imprescindível discorrer sobre as fases gradativas dessas aplicações de 
estratégias. Em fase inicial, serão preservadas as funções pouco prejudicadas do indivíduo a fim de motivá-lo e contar com seu apoio para as metas da fase intermediaria. Nessa fase inicial é elementar o aprofundamento no estudo da patologia, lesões ou dificuldade específica apresentada, elencando quais os materiais necessários, técnicas e estratégias de intervenção. É de suma importância que suas habilidades e competências sejam respeitadas, discutindo as metas desejadas e quais submetas precisam ser estabelecidas para se chegar ao objetivo final. A dificuldade das tarefas dependerá do nível de compreensão do indivíduo.

Em fase intermediária, como discorrido por Russo (2015), utilizam-se estratégias relacionadas aos processos de compreensão-retenção e recuperação-utilização. Nessa fase pode-se utilizar jogos competitivos e não-competitivos, softwares educativos, técnicas que abarquem linguagem oral, escrita, pesquisa estruturada, entre outros. Por fim, em fase final de intervenção, reavalia-se o quadro encontrado e a obtenção das metas; caso seja positivo, procede-se à alta. Caso ainda existam situações a serem trabalhadas, um novo relatório sinalizando os ganhos do paciente será elaborado. A posteriori, apresenta-se um novo plano de intervenção detalhando as etapas subsequentes do trabalho, e subsequente construção das estratégias.

Dessa maneira, intervenções de cunho neuropsicológico concernentes à atenção em crianças com TDAH têm resultado em melhoras significativas em relação à vigilância, atenção dividida e flexibilidade cognitiva (CANTIERA, 2014). Segundo Tucha et al. (2011), programas de treinamento com o tempo de cerca de oito horas apresentaram efeitos positivos no quesito da atenção, tornando-se uma possibilidade eficaz, promissora e barata de tratamento nãofarmacológico que poderá ser realizado juntamente ao uso de medicamentos. Gualtieri e Johson (2008) irão reiterar os achados de Tucha et al. (2011) ao sinalizarem que os medicamentos para o transtorno se fazem efetivos, mas que não anulam a necessidade de um acompanhamento/intervenção educacional adicional, mesmo em face de um tratamento medicamentoso bem-sucedido.

Em um estudo brasileiro sobre implementação de um guia de manejo comportamental em uma classe de $2^{\circ}$ ano regular aplicado por um professor treinado para aplicação da técnica, verificou-se que crianças que foram remanejadas quanto ao comportamento de falta de atenção e hiperatividade (Grupo Experimental - GE) tiveram redução significativa em relação ao Grupo Controle (GC) (SIQUEIRA et al., 2019). O guia de orientação para manejo comportamental de crianças e adolescentes com sinais de desatenção e hiperatividade foi proposto por Araújo 
(2012) em sua tese de doutorado, baseando-se na teoria da análise do comportamento com estratégias de controle de estímulos e uso de reforçadores. O pesquisador conclui em sua tese que: “...incorporar estratégias no planejamento escolar que visem auxiliar o professor a lidar com dificuldades específicas de seus alunos é fundamental para que a escola possa desenvolver ao máximo o potencial de aprendizagem deles.” (ARAÚJO, 2012. p. 154).

Pfiffiner et al. (2018) reitera que estratégias de intervenção focadas em crianças que portam sintomas associados ao déficit de atenção e hiperatividade possuem eficácia relevante mesmo após periodo de aplicação, ressoando em efeitos benéficos, inclusive, no ano posterior à intervenção, melhorando de forma considerável a qualidade de vida.

\section{CONCLUSÃO}

Estudos na área de Neurociências têm avançado de forma significativa nos últimos anos, permitindo conhecer de forma mais ampla o funcionamento cognitivo em indivíduos com TDAH. Os instrumentos neuropsicopedagógicos, assim como os exames neurofisiológicos, podem ser expoentes aliados na investigação dos distúrbios da atenção e nos comportamentos hiperativos. Entretanto, é importante ressaltar que nenhum diagnóstico pode ser realizado embasado somente em tais testes/instrumentos. Uma anamnese bem realizada, uma avaliação clínica criteriosa, assim como um parecer de outras especialidades (fonoaudiologia, neuropsicologia, psicopedagogia, neuropediatria entre outros), mostram-se substancialmente importantes para em conjunto elucidar a problemática, e traçar estratégias mais assertivas de investigação e intervenção neuropsicopedagógica.

Sendo assim, salienta-se a importância de conhecer e desmistificar o TDAH, levandose em conta que é, hoje, um dos principais problemas clínicos encontrados, e que atinge crianças, adolescentes e adultos. Tal problemática tem causado um grande impacto na sociedade em geral, principalmente em quem sofre com esse transtorno. Dessa maneira, a Neuropsicopedagogia tem se instituído como uma ciência teórica e metodológica que vem reverberando de forma positiva na qualidade de vida do indivíduo portador de TDAH.

Por se tratar de uma ciência transdisciplinar, abre caminhos múltiplos para se refletir como o cérebro processa as informações e de que maneira o indivíduo pode aprender a adotar novos comportamentos e formas de aprendizagens, repercutindo em uma melhora significativa nos sintomas mais expoentes. 

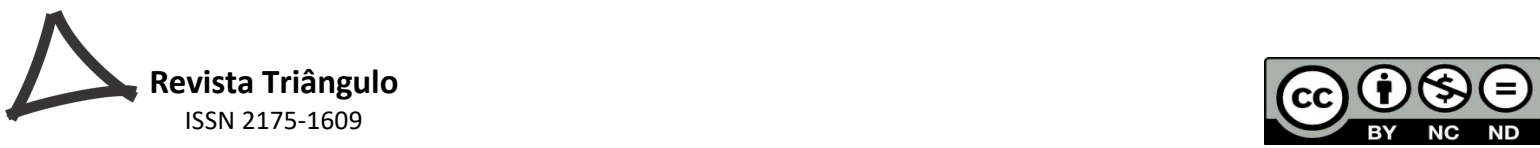

Vale ressaltar a necessidade de mais estudos sistematizados na área de Neuropsicopedagogia Clínica, principalmente no que tange a área prática e interventiva em relação ao TDAH, já que foi percebida uma dificuldade em encontrar bibliografia que correlacionasse esses dois eixos. Novos estudos poderão demonstrar de forma mais tangível a efetividade das intervenções neuropsicopedagógicas, e a identificação de procedimentos e suas respostas a essas intervenções.

\section{REFERÊNCIAS}

ASSOCIAÇÃO AMERICANA DE PSIQUIATRIA. Manual de diagnóstico e estatística de distúrbios mentais - DSM. 3 ed. - Revisão. Tradução Lúcia Helena Siqueira Barbosa; revisão técnica Sylvio Giordano Júnior. São Paulo: Manole, 1989.

ASSOCIAÇÃO AMERICANA DE PSIQUIATRIA. Manual diagnóstico e estatístico de transtornos mentais - DSM. 4 ed. - Texto Revisado. Tradução Claudia Dornelles. Porto Alegre: Artes Médicas, 2002.

ASSOCIAÇÃO AMERICANA DE PSIQUIATRIA. Manual diagnóstico e estatístico de transtornos mentais- DSM. 5 ed. Porto Alegre: Artmed, 2014.

ARAÚJO, Marcos Vinícius de. Manejo comportamental pelo professor no contexto de sala de aula de alunos identificados com TDAH: desenvolvimento, implementação e avaliação de guia de intervenção. 2012. 237 f. Tese (Doutorado em Psicologia) - Universidade Presbiteriana Mackenzie, São Paulo, 2012.

BRIDI FILHO, César Augusto; BRIDI, Fabiane Romano de Souza. Sobre o aprender e suas relações: interfaces entre neurologia, psicologia e psicopedagogia. In: ROTTA, Newra Tellechea; BRIDI FILHO, César Augusto; BRIDI, Fabiane Romano de Souza (Orgs). Neurologia e aprendizagem: abordagem multidisciplinar. São Paulo, Artmed: 2016. p. 17-28. CANTIERE, Carla Nunes. Intervenção neuropsicológica para desenvolvimento de habilidades de atenção e flexibilidade cognitiva em crianças com TDAH. 2014. Dissertação (Mestrado em Distúrbios do Desenvolvimento) - Universidade Presbiteriana Mackenzie, São Paulo, 2014.

CARVALHO, Guilherme Azevedo. O atendimento neuropsicopedagogico clínico no TDAH. Revista Científica Multidisciplinar Núcleo do Conhecimento. Ano 03, Ed. 11, Vol. 01, pp. 135-146 Nov. de 2018. ISSN:2448-0959

CERVO, Amado Luiz; BERVIAN, Pedro Alcino; DA SILVA, Roberto. Metodologia científica. 6. ed. São Paulo: Pearson Prentice Hall, 2007.

CLEMENTS, Sam. D. Minimal Brain Dysfunction in Children. U.S. Depart. of Health, Education and Welfare, 1966.

COSENZA, Ramon; GUERRA, Leonor B. Neurociência e educação: como o cérebro aprende. Porto Alegre: Artmed, 2011.

FONSECA, Vitor da. Papel das funções cognitivas, conativas e executivas na aprendizagem: uma abordagem neuropsicopedagógica. Rev. psicopedag., São Paulo, v. 31, n. 96, p. 236- 
253, 2014.

Disponível

em

$<$ http://pepsic.bvsalud.org/scielo.php?script=sci_arttext\&pid=S0103-

84862014000300002\&lng=pt\&nrm=iso $>$. acessos em 06 abr. 2021.

FÜLLE, Angelita; CARDOSO, Fabricio Bruno; RUSSO, Rita. M. T.; ROSA, Barbara M. Heck da. Neuropsicopedagogia: ciência da aprendizagem. In: RUSSO, Rita M. Toler. (Org). Neuropsicopedagogia Institucional. Curitiba: Juruá, 2018. p. 25-34.

GIL, Antonio Carlos. Métodos e técnicas de pesquisa social. 5. ed. São Paulo: Atlas, 1999. GUALTIERI, C. Tomas; JOHNSON, Linda G. Medications Do Not Necessarily Normalize Cognition in ADHD Patients. Journal of Attention Disorders, 2008.

KANDEL, Eric Richard. Em busca da memória. São Paulo: Companhia das Letras, 2009. PEREIRA, Heloisa S.; ARAUJO, Alexandra P. Q. C.; MATTOS, Paulo. Transtorno do déficit de atenção e hiperatividade (TDAH): aspectos relacionados à comorbidade com distúrbios da atividade motora. Rev. Bras. Saude Mater. Infant., Recife, v. 5, n. 4, p. 391-402, Dez. 2005. Disponível em: $\quad<$ http://www.scielo.br/scielo.php?script=sci_arttext\&pid=S1519$38292005000400002 \& \operatorname{lng}=\mathrm{en} \& \mathrm{nrm}=$ iso $>$.

Acesso em 05 Abr. 2021. https://doi.org/10.1590/S1519-38292005000400002.

PFIFFNER, Linda J.; ROONEY, Mary E.; JIANG, Yuanyuan; HAACK, Lauren M.; BEAULIEU, Allyson; MCBURNETT, Keith. Sustained effects of collaborative school-home intervention for Attention-Deficit/Hyperactivity Disorder symptoms and impairment. Acad Child Adolesc Psychiatry, v. 57, n.4, p. 245-251, 2018. Disponível em: https://www.sciencedirect.com/science/article/abs/pii/S0890856718300546. Acesso em 12 Abr. 2021. doi: 10.1016/j.jaac.2018.01.016

PORTILHO, Evelise M. Labatut.; KUSTER, Sonia M. Gomes. Estratégias de aprendizagem da criança em processo de alfabetização. Revista de Psicopedagogia, v.23, n.70, p. 23-29, 2006. ROTTA, Newra Tellechea. Transtorno de déficit de atenção/hiperatividade: aspectos clínicos. In: ROTTA, Newra; OHWEILER, Ligia; RIESGO, Rudimar dos S. (Org). Transtornos da Aprendizagem: abordagem neurobiológica e multidisciplinar. São Paulo: Artmed, 2016. p. 274-286.

RUSSO, Rita Margarida Toler. Neuropsicopedagogia Clínica: Introdução, Conceitos, Teoria e Prática. Curitiba: Juruá, 2015.

SIQUEIRA, Alisson Rogério C. de et al. Efeitos de intervenção comportamental em contexto escolar sobre desatenção e hiperatividade. Psicol. teor. prat., São Paulo, v. 21, n. 1, p. 102 118, Apr. 2019. Disponível

em:

$<$ http://pepsic.bvsalud.org/scielo.php?script=sci_arttext\&pid=S1516-

$36872019000100005 \& \operatorname{lng}=$ en\&nrm $=$ iso $>$.

Acesso em 14 Abr. 2021. http://dx.doi.org/10.5935/1980-6906/psicologia.v21n1p102-118.

TEIXEIRA, Ricardo Antonio Gonçalves. Educação do anormal a partir dos testes de inteligência. Hist. Educ., Santa Maria, v. 23, e90024, 2019. Disponível em: $<$ http://www.scielo.br/scielo.php?script=sci_arttext\&pid=S2236-

34592019000100443\&lng=en\&nrm=iso $>$. Acesso em 25 Jan. 2021. Epub Nov 25, 2019. https://doi.org/10.1590/2236-3459/90024. 
TUCHA, O.; TUCHA, L.; KAUMANN, G.; KONIG, S., LANGE, K. M., STASIK, D.; STREATHER, Z.; ENGELSCHALK, T.; LANGE, K.W. Training of attention functions in children with attention deficit hyperactivity disorder, Attention Deficit and Hiperactivity Disorder. Atten Defic Hyperact Disord. v.3, p.271-283, 2011. Disponível em: https://www.ncbi.nlm.nih.gov/pmc/articles/PMC3158847/. Acesso em 14 abr. 2021. 